\title{
Dietary Habits in Japanese Patients with Alopecia Areata
}

\author{
Teppei Hagino ${ }^{1,2}$ \\ Shizuka Okazaki ${ }^{1}$ \\ Naotaka Serizawa' \\ Kaori Suzuki' \\ Mio Kaga ${ }^{2}$ \\ Yohei Otsuka ${ }^{2}$ \\ Erina Mikami \\ Toshihiko Hoashi ${ }^{2}$ \\ Hidehisa Saeki (iD ${ }^{2}$ \\ Hiroki Matsuda ${ }^{3}$ \\ Hiroshi Mitsui ${ }^{3}$ \\ Naoko Kanda (iD) \\ 'Department of Dermatology, Nippon \\ Medical School Chiba Hokusoh Hospital, \\ Inzai, Chiba, Japan; ${ }^{2}$ Department of \\ Dermatology, Nippon Medical School, \\ Tokyo, Japan; ${ }^{3}$ Department of \\ Dermatology, Tokyo Teishin Hospital, \\ Tokyo, Japan
}

Purpose: Alopecia areata (AA) is characterized by non-scarring, patchy hair loss caused by autoimmune reactions to anagen hair follicles. The pathogenesis of AA may be affected by the diet. However, the dietary habits of patients with AA have not been precisely examined. Therefore, the aim of this study was to investigate the dietary habits of patients with AA in comparison to those of healthy controls.

Patients and Methods: We evaluated the dietary habits of 70 adult Japanese patients with AA using a brief-type self-administered diet history questionnaire and compared them to the habits of age- and sex-matched healthy controls.

Results: Japanese patients with AA had a higher body mass index (BMI) and higher intakes of vitamin $\mathrm{C}$ and fruit than the controls. Logistic regression analysis showed that AA was associated with BMI. Retinol intake was positively correlated with severity of alopecia tool (SALT) score, and linear regression analysis revealed that retinol intake was a predictor of SALT score. Retinol intake among patients with moderate to severe AA (ie, a SALT score $>25$ ) was higher than that in patients with mild AA (a SALT score $\leq 25$ ). The mean age of AA patients with atopic dermatitis (AD) was lower than that of AA patients without $A D$; however, there were no differences in nutrient or food intake between these two groups. Logistic regression analysis showed that the comorbidity AD was negatively associated with age.

Conclusion: AA was associated with a high BMI, and high retinol intake was a predictor of SALT score. Further studies should be conducted to clarify whether dietary intervention to reduce BMI or limit retinol intake can alter the development or severity of AA.

Keywords: retinol, vitamin $\mathrm{C}$, body mass index, atopic dermatitis

\section{Introduction}

Alopecia areata (AA) is an autoimmune disease characterized by patchy, nonscarring hair loss on the scalp, face, and sometimes other body areas. ${ }^{1}$ Certain triggers, such as microtrauma, can induce resident skin $\mathrm{T}$ cells to produce interferon (IFN) $-\gamma$, which induces the expression of major histocompatibility complex (MHC) class I and II molecules and interleukin (IL)-15 in the follicular epithelium. ${ }^{1}$ The hair follicles are then infiltrated by cytotoxic effector CD8+ T cells expressing natural killer group 2, member D (NKG2D), which depends on the survival cytokine IL-15. The hair follicles are also infiltrated by effector CD4+ NKG2D+ T cells expressing IFN- $\gamma$, which induces the follicular epithelium to produce IL-15 and the chemokines CXCL9/10, thus recruiting effector $\mathrm{T}$ cells bearing CXCR3. Then, autoreactive cytotoxic CD8 + NKG2D + IFN- $\gamma$-producing T cells attack anagen hair follicles via granzymes. Although no specific target autoantigens for these
Correspondence: Naoko Kanda Department of Dermatology, Nippon Medical School Chiba Hokusoh Hospital, 17I5 Kamagari, Inzai, Chiba, 270-1694 Japan

Tel +8I 47699 IIII

Fax +81476991909

Email n-kanda@nms.ac.jp 
attacking cells have been identified, they may recognize putative hair follicle autoantigens presented by MHC class I molecules. ${ }^{1}$ The ligands of NKG2D include MHC class I chain-related gene A (MICA) and cytomegalovirus UL16-binding protein 3 , and expression of both of these ligands is upregulated in lesional hair follicles. ${ }^{1}$ Type 17 helper $\mathrm{T}$ (Th17) cells that abundantly produce IL-17A infiltrate the area around the hair follicles in AA lesions and play a supporting role for cytotoxic effector cells. ${ }^{2}$ The pathogenesis of AA also involves a decrease in the number or activity of regulatory $\mathrm{T}$ cells (Tregs), which suppress effector cells. ${ }^{1,3}$

Recently, the involvement of Th2 responses was suggested in the pathogenesis of AA, and the expression levels of the Th2 cytokines IL-4, IL-5, and IL-13 were shown to be increased in the lesional skin or serum of patients with AA. ${ }^{4}$ Alopecia areata is frequently associated with atopic dermatitis (AD), a Th2-enhanced allergic disease. ${ }^{4}$ Treatment of patients with AD using dupilumab, an anti-IL-4 receptor $\alpha$ antibody, was shown to improve concomitant $\mathrm{AA},{ }^{5}$ indicating a supportive role for Th2 cytokines in the pathogenesis of AA.

Various genetic and environmental factors, including diet, are associated with the development and exacerbation of AA. Diet may modulate the pathogenesis of AA by altering hair follicles and immune responses. ${ }^{6}$ It was suggested that a Western diet, with high levels of fat, sugar, and salt and low fiber, may promote the development and/ or increase the severity of autoimmune diseases by inducing Th1/Th17 responses, suppressing Treg activity, or dysregulating the gut microbiota. ${ }^{7}$ Severe protein deficiency, delayed or skipped breakfast, or high intake of mercury-rich fish may also trigger AA. ${ }^{8}$ Deficiency of micronutrients, such as vitamins or minerals, may promote the development of $\mathrm{AA}^{6}{ }^{6}$ as serum levels of vitamin $\mathrm{D}$, zinc, and folate tended to be lower in patients with AA than in healthy controls. Some studies have suggested that too much or too little dietary vitamin A may favor the onset or exacerbation of AA. ${ }^{6,9}$ Interestingly, switching patients with celiac disease to a gluten-free diet improved concomitant AA. ${ }^{8}$ Dietary changes can also alter the composition of the gut microbiota, which modulates the immune system via the production of various microbial metabolites, such as short-chain fatty acids, that induce Tregs. Treatment of Clostridium difficile colitis in patients with AA with fecal microbiota transplantation resulted in hair growth after improvement of gut dysbiosis, ${ }^{10}$ implying the involvement of the gut microbiota in AA. Although these previous studies suggest a role for diet in the pathogenesis of AA, the dietary habits of patients with AA have not been precisely examined.

Herein, we investigated the dietary habits of adult Japanese patients with AA and compared them to those of age- and sex-matched healthy controls using a brief-type self-administered diet history questionnaire (BDHQ). ${ }^{11} \mathrm{We}$ also examined the relationship between dietary habits and disease severity and the association with $\mathrm{AD}$.

\section{Patients and Methods Study Population}

This study was conducted in accordance with the Declaration of Helsinki (2004) and was approved by the ethics committee of Nippon Medical School Chiba Hokusoh Hospital. All study participants provided written informed consent. Seventy adult ( $\geq 18$ years) Japanese patients with AA (19 men and 51 women) were recruited to participate in this study. These patients were seen in the dermatological departments of outpatient clinics. The diagnosis of AA was made clinically according to the guidelines of the National Alopecia Areata Foundation. ${ }^{12}$ Disease severity was evaluated based on the severity of alopecia tool (SALT) score. ${ }^{13}$ Fifty-six patients were treated with topical corticosteroids, nine patients were treated with oral corticosteroids, 12 patients were treated with intravenous methylprednisolone $(500 \mathrm{mg} /$ day for 3 consecutive days; steroid minipulse therapy), 10 patients were treated with excimer light, one patient was treated with narrow-band UVB, five patients were treated with topical injection of triamcinolone acetonide, and five patients were treated with regional immunotherapy using diphenylcyclopropenone. Of the 70 patients with AA, six had Hashimoto's thyroiditis, four had Basedow's disease, and two had ulcerative colitis. Seventy age- and sexmatched healthy subjects (19 men and 51 women) were included as controls. The patients and controls were living in Tokyo or Chiba. Body mass index (BMI) was calculated as the current body weight $(\mathrm{kg})$ divided by the square of the body height (m).

\section{Dietary Assessment}

The dietary habits of the patients and controls were assessed using the BDHQ, a questionnaire regarding diet during the past month (120420_BDHQ_2012.pdf (ebnjapan.org)). ${ }^{11}$ Estimates of the intake of food items, energy, and nutrients were calculated using an ad hoc 
computer algorithm for the BDHQ, based on Standard Tables of Food Composition in Japan.

\section{Statistical Analysis}

All statistical analyses were performed using EZR (Saitama Medical Center, Jichi Medical University, Saitama, Japan). ${ }^{14}$ The Shapiro-Wilk test was used to assess the normality of the data distribution. Results are expressed as the mean \pm standard deviation for variables with normal distribution or as median and as the interquartile range for variables with non-parametric distribution. Differences between patients with $\mathrm{AA}$ and the controls were analyzed by paired $t$-test for variables with normal distribution or by Wilcoxon signed-rank test for variables with non-parametric distribution. Patients with AA were divided into two groups, the moderate to severe AA group $(\mathrm{n}=16)$ with SALT scores $>25$ and the mild AA group $(n=54)$ with SALT scores $\leq 25$. A SALT score of 25 is the point at which steroid minipulse therapy is considered as a treatment option in the AA guidelines published by the Japanese Dermatological Association. ${ }^{15}$ Patients with AA were also grouped according to whether AA was associated with $\mathrm{AD}(\mathrm{n}=16)$ or without $(\mathrm{n}=54)$. Atopic dermatitis was diagnosed according to the guidelines of the Japanese Dermatological Association. ${ }^{16}$ The differences between the mild and moderate to severe AA groups and between the groups with and without $\mathrm{AD}$ were analyzed by Student's $t$-test for variables with normal distributions or by the Mann-Whitney $U$-test for variables with non-parametric distributions. Fisher's exact test was used to assess the significance of differences in the frequency distributions.

In patients with AA, the correlation between SALT score and each variable was evaluated using Spearman's rho correlation coefficients. Statistical significance was set at $P<0.05$. The predictive factors for SALT score were analyzed using linear multivariate regression analysis. The analysis included only variables with a $p$ value $<0.05$ in the univariate analyses and was adjusted for age, sex, and BMI.

The association of each variable with $\mathrm{AA}$ or $\mathrm{AD}$ comorbidity was analyzed using bivariate and multivariate logistic regression analyses. These analyses included only the variables with a $p$ value $<0.05$ in univariate analyses, adjusted for age, sex, and BMI. To avoid multicollinearity, variables with a variance inflation factor $>10$ were excluded.

\section{Results}

Comparison of the Dietary Habits Between Patients with AA and Healthy Controls

Patients with AA had higher BMIs than the controls (Table 1). The patients also had higher intake of both vitamin $\mathrm{C}$ and fruit than the controls. The alcohol intake in patients with AA appeared to be lower than that in the controls; however, the difference was not significant. According to the bivariate and multivariable logistic regression analyses (Table 2), AA was associated with a high BMI (odds ratio, 1.15; 95\% confidence interval, 1.02-1.29; $p=$ 0.0207). However, there was no significant association between $\mathrm{AA}$ and either vitamin $\mathrm{C}$ or fruit intake.

\section{Correlation Studies}

For the 70 patients with AA, the median (interquartile range) SALT score was $6.0(2.25-18.75)$, and there was no difference in SALT scores between male (6.0 [2.532.0], $\mathrm{n}=19)$ and female patients (6.0 [2.5-17.5], $\mathrm{n}=$ $51 ; p=0.953$, by Mann-Whitney $U$-test). Retinol intake was significantly correlated with SALT score (Table 3). In the linear multivariate regression analysis (Table 4), SALT score was predicted by high intake of retinol ( $\beta=40.82$, $t=2.214, p=0.0303$ ).

\section{Comparison of the Groups with Mild and Moderate to Severe AA}

There were no differences in sex ratio, age, or BMI between the mild and moderate to severe AA groups (Table 5). Retinol intake in the moderate to severe AA group was higher than that in the mild AA group.

\section{Comparison of the AA Groups with and without $A D$}

The mean age in the AA group with $\mathrm{AD}$ was lower than that in the AA group without AD (Table 6). However, there were no differences in sex ratio, BMI, SALT score, or the intake of nutrients/foods between the two groups. Meat intake in the AA group with $\mathrm{AD}$ appeared to be higher than that in the AA group without $\mathrm{AD}$, however, the difference was not significant. Logistic regression analysis revealed that the comorbidity of $\mathrm{AD}$ in patients with AA was negatively associated with age (odds ratio, 0.941; 95\% confidence interval, $0.901-0.984 ; p=0.00743$; Table 7). 
Table I Demographic Characteristics and Intake of Nutrients and Foods in Controls and Patients with Alopecia Areata (AA)

\begin{tabular}{|c|c|c|c|}
\hline & Control $(n=70)$ & AA $(n=70)$ & $p$ values \\
\hline \multirow[t]{2}{*}{ Sex } & Male 19 & Male 19 & \multirow[t]{2}{*}{$I^{c}$} \\
\hline & Female $5 \mathrm{I}$ & Female $5 \mathrm{I}$ & \\
\hline Age (years) ${ }^{\mathrm{a}}$ & $49.0(35.25-61.75)$ & $47.5(35.5-58.75)$ & 0.144 \\
\hline Body mass index $\left(\mathrm{kg} / \mathrm{m}^{2}\right)^{\mathrm{a}}$ & $20.22(19.21-22.38)$ & $21.86(20.0-24.17)$ & $0.00506 * *$ \\
\hline Energy intake $(\mathrm{kcal} / \mathrm{day})^{\mathrm{a}}$ & $1778(|46|-2010)$ & $1602(1305-2005)$ & 0.287 \\
\hline \multicolumn{4}{|l|}{ Nutrients } \\
\hline Animal protein $(\% \text { energy })^{a}$ & $8.746(6.625-10.009)$ & $8.823(7.165-10.284)$ & 0.730 \\
\hline Vegetable protein $(\% \text { energy) })^{\mathrm{b}}$ & $6.50 \pm 1.03$ & $6.44 \pm 1.19$ & 0.687 \\
\hline Animal fat $(\% \text { energy })^{\mathrm{a}}$ & $12.69(10.76-15.90)$ & $13.25(9.16-15.54)$ & 0.314 \\
\hline Vegetable fat (\% energy) ${ }^{\mathrm{a}}$ & $15.10 \pm 30.37$ & $15.47 \pm 3.89$ & 0.503 \\
\hline Carbohydrate (\% energy) ${ }^{\mathrm{b}}$ & $50.45 \pm 7.40$ & $51.45 \pm 8.44$ & 0.467 \\
\hline $\mathrm{Na}(\mathrm{mg} / \mathrm{kcal})^{\mathrm{a}}$ & $2.13(1.97-2.56)$ & $2.27(2.05-2.65)$ & 0.377 \\
\hline $\mathrm{K}(\mathrm{mg} / \mathrm{kcal})^{\mathrm{a}}$ & $1.35(1.10-1.69)$ & $1.29(1.13-1.62)$ & 0.861 \\
\hline $\mathrm{Ca}(\mu \mathrm{g} / \mathrm{kcal})^{\mathrm{a}}$ & $283.0(231 . I-346.0)$ & $272.0(213.5-347.5)$ & 0.748 \\
\hline $\mathrm{Mg}(\mu \mathrm{g} / \mathrm{kcal})^{\mathrm{a}}$ & $133.5(114.5-160.8)$ & $127.2(\mid 10.1-158.2)$ & 0.472 \\
\hline $\mathrm{Pi}(\mu \mathrm{g} / \mathrm{kcal})^{\mathrm{a}}$ & $549.5(488.6-642.1)$ & $552.2(462.8-652.5)$ & 0.627 \\
\hline $\mathrm{Fe}(\mu \mathrm{g} / \mathrm{kcal})^{\mathrm{a}}$ & $4.27(3.43-5.07)$ & $4.08(3.4 \mathrm{I}-5.04)$ & 0.704 \\
\hline $\mathrm{Zn}(\mu \mathrm{g} / \mathrm{kcal})^{\mathrm{a}}$ & $4.41(3.94-4.85)$ & $4.35(3.86-4.93)$ & 0.386 \\
\hline $\mathrm{Cu}(\mu \mathrm{g} / \mathrm{kcal})^{\mathrm{a}}$ & $0.603(0.521-0.670)$ & $0.582(0.513-0.676)$ & 0.874 \\
\hline $\operatorname{Mn}(\mu \mathrm{g} / \mathrm{kcal})^{\mathrm{a}}$ & $1.65(1.41-1.97)$ & $1.64(1.34-2.38)$ & 0.311 \\
\hline Retinol $(\mu \mathrm{g} / \mathrm{kcal})^{\mathrm{a}}$ & $0.256(0.139-0.370)$ & $0.174(0.137-0.293)$ & 0.114 \\
\hline$\beta$-carotene $(\mu \mathrm{g} / \mathrm{kcal})^{\mathrm{a}}$ & $1.76(1.03-2.95)$ & $1.92(1.14-3.06)$ & 0.464 \\
\hline 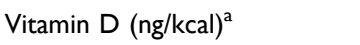 & $5.18(3.47-7.77)$ & $5.49(3.55-8.52)$ & 0.953 \\
\hline$\alpha$-tocopherol $(\mu \mathrm{g} / \mathrm{kcal})^{\mathrm{b}}$ & $4.145 \pm 1.009$ & $4.350 \pm 1.093$ & 0.178 \\
\hline Vitamin $\mathrm{K}(\mu \mathrm{g} / \mathrm{kcal})^{\mathrm{a}}$ & $0.165(0.116-0.220)$ & $0.137(0.105-0.216)$ & 0.364 \\
\hline Vitamin $\mathrm{BI}(\mu \mathrm{g} / \mathrm{kcal})^{\mathrm{a}}$ & $0.419(0.369-0.499)$ & $0.420(0.349-0.514)$ & 0.520 \\
\hline Vitamin B2 $(\mu \mathrm{g} / \mathrm{kcal})^{\mathrm{a}}$ & $0.730(0.634-0.863)$ & $0.716(0.591-0.865)$ & 0.911 \\
\hline Niacin $(\mu \mathrm{g} / \mathrm{kcal})^{\mathrm{a}}$ & $9.53(8.01-10.88)$ & $9.48(8.03-11.14)$ & 0.497 \\
\hline Vitamin B6 $(\mu \mathrm{g} / \mathrm{kcal})^{\mathrm{a}}$ & $0.676(0.587-0.805)$ & $0.690(0.53 I-0.827)$ & 0.547 \\
\hline Vitamin BI2 (ng/kcal) ${ }^{\mathrm{a}}$ & $3.94(2.98-5.77)$ & $3.96(3.17-5.84)$ & 0.986 \\
\hline Foric acid $(\mu \mathrm{g} / \mathrm{kcal})^{\mathrm{a}}$ & $0.176(0.142-0.237)$ & $0.172(0.147-0.232)$ & 0.865 \\
\hline Panthotenic acid $(\mu \mathrm{g} / \mathrm{kcal})^{\mathrm{a}}$ & $3.62(3.05-4.11)$ & $3.52(3.00-4.03)$ & 0.717 \\
\hline Vitamin C $(\mu \mathrm{g} / \mathrm{kcal})^{\mathrm{a}}$ & $55.3(40.8-77.9)$ & $61.1(49.2-88.3)$ & $0.0211^{*}$ \\
\hline
\end{tabular}


Table I (Continued).

\begin{tabular}{|c|c|c|c|}
\hline & Control $(n=70)$ & AA $(n=70)$ & $p$ values \\
\hline \multirow[t]{2}{*}{ Sex } & Male 19 & Male 19 & \multirow[t]{2}{*}{$I^{c}$} \\
\hline & Female $5 \mathrm{I}$ & Female $5 \mathrm{I}$ & \\
\hline SFA (\% energy) $)^{\mathrm{b}}$ & $8.016 \pm 2.134$ & $7.596 \pm 2.083$ & 0.216 \\
\hline MUFA (\% energy) ${ }^{\mathrm{b}}$ & $10.298 \pm 2.082$ & $10.126 \pm 2.370$ & 0.594 \\
\hline n-3PUFA(\% energy) ${ }^{a}$ & $1.21(1.06-1.45)$ & $1.26(1.03-1.55)$ & 0.673 \\
\hline n-6PUFA(\% energy) ${ }^{b}$ & $5.302 \pm 1.046$ & $5.509 \pm 1.287$ & 0.274 \\
\hline Cholesterol $(\mu \mathrm{g} / \mathrm{kcal})^{\mathrm{a}}$ & 207.7 (I64.6-250.9) & $211.3(168.8-247.4)$ & 0.704 \\
\hline Dietary fiber $(\mathrm{mg} / \mathrm{kcal})^{\mathrm{a}}$ & $6.281(5.19-8.12)$ & $6.161(4.80-7.85)$ & 0.874 \\
\hline Alcohol (\% energy) ${ }^{\mathrm{a}}$ & $1.886(0.105-7.595)$ & $0.0182(0-3.009)$ & 0.058 \\
\hline \multicolumn{4}{|l|}{ Foods } \\
\hline Cereals (mg/kcal) ${ }^{\mathrm{b}}$ & $183.90 \pm 67.15$ & $186.44 \pm 64.89$ & 0.803 \\
\hline Potatoes (mg/kcal) & 13.25 (7.72-3।.59) & $16.34(9.78-34.53)$ & 0.824 \\
\hline Pulses $(\mathrm{mg} / \mathrm{kcal})^{\mathrm{a}}$ & $1.80(1.07-3.17)$ & $2.02(1.30-4.60)$ & 0.093 \\
\hline $\begin{array}{l}\text { Green and yellow vegetables }(\mathrm{mg} / \\
\mathrm{kcal})^{\mathrm{a}}\end{array}$ & 59.31 (34.76-83.83) & $56.33(30.60-90.18)$ & 0.949 \\
\hline Other vegetables $(\mathrm{mg} / \mathrm{kcal})^{\mathrm{a}}$ & 75.54 (54.64-I05.52) & 87.86 (57.44-22.99) & 0.512 \\
\hline Fruit $(\mathrm{mg} / \mathrm{kcal})^{\mathrm{a}}$ & $50.65(28.56-86.9 I)$ & $67.59(23.39-115.67)$ & $0.0313^{*}$ \\
\hline Fish and shellfish $(\mathrm{mg} / \mathrm{kcal})^{\mathrm{a}}$ & 29.85 (20.49-47.44) & $30.06(22.23-44.76)$ & 0.842 \\
\hline Meat $(\mathrm{mg} / \mathrm{kcal})^{\mathrm{a}}$ & $46.29(36.10-57.04)$ & $43.32(29.80-57.64)$ & 0.109 \\
\hline Eggs $(\mathrm{mg} / \mathrm{kcal})^{\mathrm{a}}$ & 15.88 (|0.9|-28.0I) & $20.83(12.42-29.54)$ & 0.505 \\
\hline Dairy products $(\mathrm{mg} / \mathrm{kcal})^{\mathrm{a}}$ & $79.58(28.40-107.61)$ & $73.32(24.59-12.70)$ & 0.939 \\
\hline Oils and fats $(\mathrm{mg} / \mathrm{kcal})^{\mathrm{b}}$ & $6.11 \pm 2.67$ & $6.63 \pm 2.93$ & 0.204 \\
\hline Confection (mg/kcal) ${ }^{\mathrm{a}}$ & 26.18 (13.02-49.69) & $25.39(|5.76-4| .72)$ & 0.819 \\
\hline Beverages $(\mathrm{mg} / \mathrm{kcal})^{\mathrm{a}}$ & $427.85(246.2-542.6)$ & $382.17(276.3-595.2)$ & 0.574 \\
\hline Seasonings and spices $(\mathrm{mg} / \mathrm{kcal})^{\mathrm{a}}$ & $101.85(76.49-142.74)$ & $124.67(86.55-73.25)$ & 0.139 \\
\hline Sugar/sweeteners $(\mathrm{mg} / \mathrm{kcal})^{\mathrm{a}}$ & $1.80(1.07-3.17)$ & $2.01(1.30-4.60)$ & 0.093 \\
\hline
\end{tabular}

Notes: a Data provided as the median (interquartile range), analyzed by Wilcoxon signed rank test. b Data provided as the mean \pm standard deviation, analyzed by paired $t$-test. 'Fisher's exact test was used to test the significance of difference in frequency distribution. $*$ Significant differences at $p<0.05$. $* *$ Significant difference at $p<0.0 \mathrm{I}$. Abbreviations: SFA, saturated fatty acid; MUFA, monounsaturated fatty acid; PUFA, polyunsaturated fatty acid.

\section{Discussion}

The BMI of patients with AA was higher than that of the controls, and AA was associated with BMI in the logistic regression analysis. The results indicate that a high BMI may be related to the development of AA. Several studies have reported that the prevalence of obesity is higher in patients with AA and other autoimmune diseases, such as systemic lupus erythematosus and rheumatoid arthritis, than in the reference group. ${ }^{17,18}$ Obesity is characterized by low levels of chronic inflammation, and the adipose tissues in obese subjects contain enlarged adipocytes that abundantly produce inflammatory adipokines, such as leptin, as well as excess infiltration of inflammatory M1 macrophages, IFN- $\gamma$-producing $\mathrm{CD} 4+$ and $\mathrm{CD} 8+\mathrm{T}$ cells, 
Table 2 The Association of Alopecia Areata with Each Variable Analyzed by Multiple Logistic Regression Analysis

\begin{tabular}{|l|c|c|c|}
\hline & Odds Ratio & 95\% Confidential Interval & P values \\
\hline (Intercept) & 0.0438 & $0.00188-1.02$ & 0.0517 \\
Age (years) & 0.986 & $0.963-1.01$ & 0.232 \\
Sex $(\mathrm{M}=\mathrm{I}, \mathrm{F}=2)$ & 1.17 & $0.512-2.66$ & 0.715 \\
Body mass index $\left(\mathrm{kg} / \mathrm{m}^{2}\right)$ & 1.15 & $1.02-1.29$ & $0.0207^{*}$ \\
Vitamin C $(\mu \mathrm{g} / \mathrm{kcal})$ & 1.01 & $0.99-1.02$ & 0.522 \\
Fruit $(\mathrm{mg} / \mathrm{kcal})$ & 1.00 & $0.995-1.01$ & 0.420 \\
\hline
\end{tabular}

Note: *Statistically significant at $p<0.05$.

Table 3 Correlations of Severity of Alopecia Tool with Intakes of Nutrients/Foods in Patients with Alopecia Areata Using Spearman Correlation Coefficients

\begin{tabular}{|c|c|c|c|c|c|}
\hline & Rho & $p$ & & Rho & $p$ \\
\hline Age (years) & 0.0435 & 0.72 & \multicolumn{3}{|l|}{ Nutrients II } \\
\hline Body mass index $\left(\mathrm{kg} / \mathrm{m}^{2}\right)$ & 0.218 & 0.07 & SFA (\% energy) & -0.0927 & 0.445 \\
\hline Energy intake (kcal/day) & -0.134 & 0.27 & MUFA (\% energy) & -0.0211 & 0.862 \\
\hline \multicolumn{3}{|l|}{ Nutrients I } & n-3PUFA (\% energy) & -0.161 & 0.182 \\
\hline Animal protein (\% energy) & -0.0408 & 0.737 & n-6PUFA (\% energy) & -0.00796 & 0.948 \\
\hline Vegetable protein (\% energy) & -0.199 & 0.0986 & Cholesterol $(\mu \mathrm{g} / \mathrm{kcal})$ & -0.0727 & 0.55 \\
\hline Animal fat (\% energy) & -0.0451 & 0.711 & Dietary fiber $(\mathrm{mg} / \mathrm{kcal})$ & -0.0628 & 0.605 \\
\hline Vegetable fat (\% energy) & $-0.044 I$ & 0.717 & Alcohol (\% energy) & 0.0711 & 0.558 \\
\hline Carbohydrate (\% energy) & -0.168 & 0.163 & \multicolumn{3}{|l|}{ Foods } \\
\hline $\mathrm{Na}(\mathrm{mg} / \mathrm{kcal})$ & 0.0624 & 0.608 & Cereals (mg/kcal) & -0.166 & 0.17 \\
\hline $\mathrm{K}$ (mg/kcal) & -0.0416 & 0.732 & Potatoes (mg/kcal) & $-0.07 I I$ & 0.559 \\
\hline $\mathrm{Ca}(\mu \mathrm{g} / \mathrm{kcal})$ & -0.0481 & 0.692 & Pulses (mg/kcal) & 0.133 & 0.273 \\
\hline $\mathrm{Mg}(\mu \mathrm{g} / \mathrm{kcal})$ & -0.0199 & 0.87 & Green and yellow vegetables (mg/kcal) & 0.0104 & 0.932 \\
\hline $\mathrm{Pi}(\mu \mathrm{g} / \mathrm{kcal})$ & -0.0812 & 0.504 & Other vegetables (mg/kcal) & -0.0384 & 0.752 \\
\hline $\mathrm{Fe}(\mu \mathrm{g} / \mathrm{kcal})$ & -0.0842 & 0.488 & Fruit (mg/kcal) & -0.124 & 0.306 \\
\hline Zn ( $\mu g / k c a l)$ & -0.172 & 0.156 & Fish and shellfish (mg/kcal) & 0.112 & 0.355 \\
\hline $\mathrm{Cu}(\mu \mathrm{g} / \mathrm{kcal})$ & -0.117 & 0.334 & Meat (mg/kcal) & -0.0605 & 0.619 \\
\hline Mn ( $\mu g / k c a l)$ & -0.104 & 0.393 & Eggs (mg/kcal) & -0.128 & 0.292 \\
\hline Retinol ( $\mu \mathrm{g} / \mathrm{kcal})$ & 0.272 & $0.0228 *$ & Dairy products (mg/kcal) & 0.0344 & 0.778 \\
\hline$\beta$-carotene $(\mu \mathrm{g} / \mathrm{kcal})$ & 0.031 & 0.799 & Oils and fats (mg/kcal) & 0.0895 & 0.461 \\
\hline Vitamin D (ng/kcal) & 0.0364 & 0.765 & Confection (mg/kcal) & 0.014 & 0.909 \\
\hline$\alpha$-tocopherol $(\mu \mathrm{g} / \mathrm{kcal})$ & -0.0315 & 0.796 & Beverages (mg/kcal) & -0.0221 & 0.856 \\
\hline Vitamin $\mathrm{K}(\mu \mathrm{g} / \mathrm{kcal})$ & -0.0552 & 0.65 & Seasonings and spices (mg/kcal) & -0.0788 & 0.516 \\
\hline Vitamin $\mathrm{BI}(\mu \mathrm{g} / \mathrm{kcal})$ & -0.204 & 0.0903 & Sugar/sweeteners (mg/kcal) & 0.133 & 0.273 \\
\hline
\end{tabular}

(Continued) 
Table 3 (Continued).

\begin{tabular}{|c|c|c|c|c|}
\hline & Rho & $p$ & Rho & $p$ \\
\hline Vitamin B2 $(\mu \mathrm{g} / \mathrm{kcal})$ & -0.0875 & 0.471 & & \\
\hline Niacin $(\mu g / k c a l)$ & 0.0365 & 0.764 & & \\
\hline Vitamin B6( $\mu \mathrm{g} / \mathrm{kcal})$ & -0.0364 & 0.765 & & \\
\hline Vitamin BI2 (ng/kcal) & 0.0984 & 0.418 & & \\
\hline Foric acid ( $\mu \mathrm{g} / \mathrm{kcal})$ & -0.00861 & 0.944 & & \\
\hline Panthotenic acid ( $\mu \mathrm{g} / \mathrm{kcal})$ & -0.0832 & 0.494 & & \\
\hline Vitamin C ( $\mu \mathrm{g} /$ kcal) & -0.087 & 0.474 & & \\
\hline
\end{tabular}

Note: *Statistically significant at $<0.05$.

Abbreviations: SFA, saturated fatty acid; MUFA, monounsaturated fatty acid; PUFA, polyunsaturated fatty acid.

Table 4 The Predictive Factors for Severity of Alopecia Tool Analyzed by Linear Multivariate Regression Analysis

\begin{tabular}{|l|c|c|c|c|}
\hline & $\boldsymbol{\beta}$ Coefficient & Standard Error & $\boldsymbol{t}$ & $\boldsymbol{p}$ \\
\hline (Intercept) & 25.33 & 25.78 & 0.983 & 0.329 \\
Age & -0.0297 & 0.214 & -0.139 & 0.890 \\
Sex & -3.97 & 7.994 & -0.497 & 0.621 \\
Body mass index $\left(\mathrm{kg} / \mathrm{m}^{2}\right)$ & -0.291 & 0.866 & -0.336 & 0.738 \\
Retinol $(\mu \mathrm{g} / \mathrm{kcal})$ & 40.82 & 18.44 & 2.214 & $0.0303 *$ \\
\hline
\end{tabular}

Note: *Statistically significant at $p<0.05$.

and Th17 cells. In addition, the production of antiinflammatory adipokines, such as adiponectin, M2 macrophages, and Tregs is decreased in the adipose tissues of obese subjects. ${ }^{19}$ These inflammatory cytokines and adipokines may be released from the adipose tissues, circulate in the blood, and reach peripheral sites. In particular, IFN$\gamma$ might induce type 1 autoimmune diseases ${ }^{18}$ and promote MHC class I/II expression in hair follicles, thus triggering AA. Leptin is an inflammatory adipokine that induces dendritic cells (DCs) to produce IL-12 and drives the polarization of naïve CD4+ T cells toward Th1 cells. ${ }^{19}$ Furthermore, the decrease in the levels of the antiinflammatory adipokine adiponectin may contribute to autoimmune inflammation. Compared to wild-type mice, adiponectin knockout mice developed worsened experimental autoimmune encephalomyelitis, with increased production of IFN- $\gamma$ and IL-17A in CD4+ T cells. ${ }^{20}$ A recent study reported that serum adiponectin levels in patients with AA were lower than those in the reference group. $^{21}$

Intakes of vitamin $\mathrm{C}$ and fruit were higher in patients with AA than in the controls; however, none of these variables were independently associated with AA in the logistic regression analysis. Since vitamin $\mathrm{C}$ is abundant in fruit, increased intake of vitamin $\mathrm{C}$ in patients with $\mathrm{AA}$ may reflect the increased intake of fruit. The present results suggest that increased intake of vitamin $\mathrm{C}$ may be related to the development of AA, which may be associated with the Th1-inducible effects of vitamin C. In a previous study, vitamin $\mathrm{C}$ induced murine DCs to produce IL-12 via activation of the p38 mitogen-activated protein kinase and then promoted their activity to drive the differentiation of naïve $\mathrm{T}$ cells into $\mathrm{Th} 1$ cells. $^{22}$ Vitamin C induced the murine DCs to secrete more IL12 and IL-15 and enhanced their ability to differentiate CD8 + T cells into IFN- $\gamma+$ cytotoxic memory T cells. ${ }^{22}$

Alternatively, patients with AA might intentionally consume larger amounts of vitamin $\mathrm{C}$ to manage their hair loss, as vitamin $\mathrm{C}$ is recommended for subjects with various types of hair loss, including AA, on websites, TV, and horizontal publications because vitamin $C$ induces the synthesis of collagen fibers, a component of hair, and promotes the intestinal absorption of iron, which is required for hair growth. ${ }^{23}$ 
Table 5 Demographic Characteristics and Intake of Nutrients and Foods in Mild (Severity of Alopecia Tool [SALT] $\leqq 25)$ and Moderate to Severe (SALT > 25) Alopecia Areata Groups

\begin{tabular}{|c|c|c|c|c|c|}
\hline \multirow{3}{*}{ Sex } & \multicolumn{2}{|c|}{ Mild $(n=54)$} & \multicolumn{2}{|c|}{ Moderate to Severe $(n=16)$} & \multirow{2}{*}{$\frac{p \text { values }}{0.752^{c}}$} \\
\hline & \multirow{2}{*}{$\begin{array}{c}\text { Male } \\
\text { Female }\end{array}$} & \multirow{2}{*}{$\begin{array}{l}14 \\
40\end{array}$} & \multirow{2}{*}{$\begin{array}{c}\text { Male } \\
\text { Female }\end{array}$} & \multirow{2}{*}{$\begin{array}{c}5 \\
I I\end{array}$} & \\
\hline & & & & & \\
\hline Age (years) ${ }^{a}$ & \multicolumn{2}{|c|}{$48.50 \pm 16.38$} & \multicolumn{2}{|c|}{$47.94 \pm 18.20$} & 0.907 \\
\hline Body mass index $\left(\mathrm{kg} / \mathrm{m}^{2}\right)^{\mathrm{b}}$ & \multicolumn{2}{|c|}{$21.93(19.54-24.40)$} & \multicolumn{2}{|c|}{$21.86(21.06-23.75)$} & 0.654 \\
\hline Energy intake $(\mathrm{kcal} / \mathrm{day})^{\mathrm{b}}$ & \multicolumn{2}{|c|}{$1606(|36|-2028)$} & \multicolumn{2}{|c|}{ I545 (I22|-|853) } & 0.390 \\
\hline \multicolumn{6}{|l|}{ Nutrients } \\
\hline Animal protein $(\% \text { energy })^{a}$ & \multicolumn{2}{|c|}{$8.578 \pm 2.786$} & \multicolumn{2}{|c|}{$8.855 \pm 4.296$} & 0.760 \\
\hline Vegetable protein $(\% \text { energy })^{a}$ & \multicolumn{2}{|c|}{$6.507 \pm 1.272$} & \multicolumn{2}{|c|}{$6.196 \pm 0.831$} & 0.362 \\
\hline Animal fat $(\% \text { energy })^{a}$ & \multicolumn{2}{|c|}{$12.77 \pm 4.00$} & \multicolumn{2}{|c|}{$13.05 \pm 5.88$} & 0.830 \\
\hline 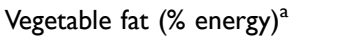 & \multicolumn{2}{|c|}{$15.51 \pm 4.02$} & \multicolumn{2}{|c|}{$15.34 \pm 3.56$} & 0.885 \\
\hline Carbohydrate $(\% \text { energy })^{a}$ & \multicolumn{2}{|c|}{$52.17 \pm 8.04$} & \multicolumn{2}{|c|}{$49.01 \pm 9.56$} & 0.190 \\
\hline $\mathrm{Na}(\mathrm{mg} / \mathrm{kcal})^{\mathrm{b}}$ & \multicolumn{2}{|c|}{$2.230(1.954-2.440)$} & \multicolumn{2}{|c|}{$2.456(2.069-2.699)$} & 0.506 \\
\hline $\mathrm{K}(\mathrm{mg} / \mathrm{kcal})^{\mathrm{b}}$ & \multicolumn{2}{|c|}{$1.302(1.133-1.635)$} & \multicolumn{2}{|c|}{$1.277(1.109-1.506)$} & 0.796 \\
\hline $\mathrm{Ca}(\mu \mathrm{g} / \mathrm{kcal})^{\mathrm{b}}$ & 273.5 & 7.2) & 257.9 & 4.5) & 0.413 \\
\hline $\mathrm{Mg}(\mu \mathrm{g} / \mathrm{kcal})^{\mathrm{b}}$ & 128.7 & I.5) & 126.2 & 6.8) & 0.883 \\
\hline $\mathrm{Pi}(\mu \mathrm{g} / \mathrm{kcal})^{\mathrm{b}}$ & 555.1 & I.6) & 522.1 & I.7) & 0.506 \\
\hline $\mathrm{Fe}(\mu \mathrm{g} / \mathrm{kcal})^{\mathrm{b}}$ & 4.05 & & 4.24 & & 0.994 \\
\hline $\mathrm{Zn}(\mu \mathrm{g} / \mathrm{kcal})^{\mathrm{a}}$ & $4.3 \varepsilon$ & & & & 0.398 \\
\hline $\mathrm{Cu}(\mu \mathrm{g} / \mathrm{kcal})^{\mathrm{a}}$ & 0.6 & & & & 0.559 \\
\hline $\operatorname{Mn}(\mu g / k c a l)^{b}$ & 1.60 & & 1.95 & & 0.711 \\
\hline Retinol $(\mu \mathrm{g} / \mathrm{kcal})$ & 0.163 & 14) & 0.310 & 12) & $0.0165 *$ \\
\hline$\beta$-carotene $(\mu \mathrm{g} / \mathrm{kcal})^{\mathrm{b}}$ & 1.88 & & 2.28 & & 0.525 \\
\hline Vitamin D (ng/kcal) ${ }^{\mathrm{b}}$ & 5.68 & & 4.69 & & 0.994 \\
\hline$\alpha$-tocopherol $(\mu \mathrm{g} / \mathrm{kcal})^{\mathrm{a}}$ & 4.35 & & & & 0.999 \\
\hline Vitamin $\mathrm{K}(\mu \mathrm{g} / \mathrm{kcal})^{\mathrm{b}}$ & 0.138 & 16) & 0.118 & & 0.610 \\
\hline Vitamin $\mathrm{BI}(\mu \mathrm{g} / \mathrm{kcal})^{\mathrm{a}}$ & 0.4 & & & & 0.363 \\
\hline Vitamin B2 $(\mu \mathrm{g} / \mathrm{kcal})^{\mathrm{b}}$ & 0.716 & 367) & 0.702 & 301) & 0.552 \\
\hline Niacin $(\mu \mathrm{g} / \mathrm{kcal})^{\mathrm{a}}$ & 9.5 & & 10.2 & & 0.342 \\
\hline Vitamin B6 $(\mu \mathrm{g} / \mathrm{kcal})^{\mathrm{a}}$ & 0.7 & & & & 0.782 \\
\hline Vitamin BI2 (ng/kcal) ${ }^{\mathrm{b}}$ & 4.10 & & 3.92 & & 0.872 \\
\hline Foric acid $(\mu \mathrm{g} / \mathrm{kcal})^{\mathrm{b}}$ & 0.167 & 32) & 0.189 & 36) & 0.742 \\
\hline Panthotenic acid $(\mu \mathrm{g} / \mathrm{kcal})^{\mathrm{b}}$ & 3.520 & $96)$ & 3.474 & 78) & 0.543 \\
\hline Vitamin C $(\mu \mathrm{g} / \mathrm{kcal})^{\mathrm{b}}$ & 61.12 & .30) & 62.92 & .33) & 0.629 \\
\hline
\end{tabular}

(Continued) 
Table 5 (Continued).

\begin{tabular}{|c|c|c|c|c|c|}
\hline \multirow{3}{*}{ Sex } & \multicolumn{2}{|c|}{ Mild $(n=54)$} & \multicolumn{2}{|c|}{ Moderate to Severe $(n=16)$} & \multirow{3}{*}{$\frac{p \text { values }}{0.752^{c}}$} \\
\hline & \multirow{2}{*}{$\begin{array}{c}\text { Male } \\
\text { Female }\end{array}$} & \multirow{2}{*}{$\begin{array}{l}14 \\
40\end{array}$} & \multirow{2}{*}{$\begin{array}{c}\text { Male } \\
\text { Female }\end{array}$} & \multirow{2}{*}{$\begin{array}{c}5 \\
I I\end{array}$} & \\
\hline & & & & & \\
\hline SFA (\% energy) $)^{a}$ & \multicolumn{2}{|c|}{$7.623 \pm 1.898$} & \multicolumn{2}{|c|}{$7.504 \pm 2.686$} & 0.843 \\
\hline MUFA (\% energy) ${ }^{a}$ & \multicolumn{2}{|c|}{$10.109 \pm 2.350$} & \multicolumn{2}{|c|}{$10.185 \pm 2.512$} & 0.911 \\
\hline n-3PUFA(\% energy) ${ }^{a}$ & \multicolumn{2}{|c|}{$1.276 \pm 0.348$} & \multicolumn{2}{|c|}{$1.400 \pm 0.46 \mathrm{I}$} & 0.250 \\
\hline n-6PUFA(\% energy) ${ }^{a}$ & \multicolumn{2}{|c|}{$5.470 \pm 1.195$} & \multicolumn{2}{|c|}{$5.643 \pm 1.596$} & 0.639 \\
\hline Cholesterol $(\mu g / \mathrm{kcal})^{\mathrm{a}}$ & \multicolumn{2}{|c|}{$214.01 \pm 71.37$} & \multicolumn{2}{|c|}{$196.83 \pm 80.60$} & 0.414 \\
\hline Dietary fiber $(\mathrm{mg} / \mathrm{kcal})^{\mathrm{b}}$ & \multicolumn{2}{|c|}{$6.11(4.80-7.92)$} & \multicolumn{2}{|c|}{$6.57(5 .||-7.3 \mid)$} & 0.939 \\
\hline Alcohol $(\% \text { energy })^{\mathrm{b}}$ & \multicolumn{2}{|c|}{$0.018(0-1.065)$} & \multicolumn{2}{|c|}{$0.454(0-7.249)$} & 0.496 \\
\hline \multicolumn{6}{|l|}{ Foods } \\
\hline Cereals (mg/kcal) ${ }^{\mathrm{a}}$ & \multicolumn{2}{|c|}{$188.30 \pm 61.55$} & \multicolumn{2}{|c|}{$180.16 \pm 76.99$} & 0.663 \\
\hline Potatoes $(\mathrm{mg} / \mathrm{kcal})^{\mathrm{b}}$ & \multicolumn{2}{|c|}{$15.36(9.92-38.02)$} & \multicolumn{2}{|c|}{$24.97(9.25-30.68)$} & 0.796 \\
\hline Pulses $(\mathrm{mg} / \mathrm{kcal})^{\mathrm{b}}$ & \multicolumn{2}{|c|}{$1.92(1.35-4.60)$} & \multicolumn{2}{|c|}{$2.78(1.28-4.57)$} & 0.711 \\
\hline $\begin{array}{l}\text { Green and yellow vegetables }(\mathrm{mg} / \\
\mathrm{kcal})^{\mathrm{b}}\end{array}$ & \multicolumn{2}{|c|}{$53.79(29.82-90.18)$} & \multicolumn{2}{|c|}{$63.23(41.17-83.14)$} & 0.732 \\
\hline Other vegetables $(\mathrm{mg} / \mathrm{kcal})^{\mathrm{a}}$ & \multicolumn{2}{|c|}{$90.284 \pm 46.788$} & \multicolumn{2}{|c|}{$85.674 \pm 43.021$} & 0.724 \\
\hline Fruit $(\mathrm{mg} / \mathrm{kcal})^{\mathrm{b}}$ & 72.41 & 93) & 35.40 & & 0.105 \\
\hline Fish and shellfish $(\mathrm{mg} / \mathrm{kcal})^{\mathrm{b}}$ & 29.30 & & 35.21 & & 0.454 \\
\hline Meat $(\mathrm{mg} / \mathrm{kcal})^{\mathrm{a}}$ & & & 45.2 & & 0.737 \\
\hline Eggs (mg/kcal) $)^{\mathrm{b}}$ & 21.13 & & 15.16 & & 0.317 \\
\hline Dairy products $(\mathrm{mg} / \mathrm{kcal})^{\mathrm{b}}$ & 74.09 & .76) & 52.80 & & 0.506 \\
\hline Oils and fats $(\mathrm{mg} / \mathrm{kcal})^{\mathrm{a}}$ & & & 7.25 & & 0.338 \\
\hline Confection (mg/kcal) ${ }^{b}$ & 26.31 & & 20.42 & & 0.543 \\
\hline Beverages $(\mathrm{mg} / \mathrm{kcal})^{\mathrm{b}}$ & 382.2 & & 400.1 & & 0.928 \\
\hline Seasonings and spices $(\mathrm{mg} / \mathrm{kcal})^{\mathrm{b}}$ & 122.6 & & 151.9 & & 0.774 \\
\hline Sugar/sweeteners $(\mathrm{mg} / \mathrm{kcal})^{\mathrm{b}}$ & 1.918 & & 2.781 & & 0.711 \\
\hline
\end{tabular}

Notes: ${ }^{a}$ Data provided as the mean \pm standard deviation, analyzed by Student's $t$-test. ${ }^{b}$ Data provided as the median (interquartile range), analyzed by Mann-Whitney $U$-test. 'Fisher's exact test was used to test the significance of difference in frequency distribution. *Significant difference at $p<0.05$.

Abbreviations: SFA, saturated fatty acid; MUFA, monounsaturated fatty acid; PUFA, polyunsaturated fatty acid.

Alcohol intake tended to be lower in patients with AA than in the controls, although the difference was not significant. The results are consistent with those of a previous study showing that alcohol consumption was associated with a decreased risk of AA. ${ }^{24}$ This finding might reflect the fact that intake of a moderate amount of alcohol (30 g/ day) decreased both the expression of nuclear factor- $\kappa \mathrm{B}$ in leukocytes and plasma levels of pro-inflammatory IL-18 and increased plasma levels of anti-inflammatory adiponectin, indicating immunosuppressive effects of moderate amount of alcohol. ${ }^{25}$

Intake of retinol, also called vitamin A1, was correlated with SALT score. This result suggests that high retinol intake may exacerbate AA via retinoic acid (RA), which is an active 
Table 6 Demographic Characteristics and Intake of Nutrients and Foods in Alopecia Areata (AA) Groups with and without Atopic Dermatitis (AD)

\begin{tabular}{|c|c|c|c|c|c|}
\hline \multirow{3}{*}{ Sex } & \multicolumn{2}{|c|}{ AA without AD $(n=54)$} & \multicolumn{2}{|c|}{ AA with AD $(n=16)$} & \multirow{2}{*}{$\frac{p \text { values }}{0.343^{c}}$} \\
\hline & \multirow{2}{*}{$\begin{array}{c}\text { Male } \\
\text { Female }\end{array}$} & \multirow{2}{*}{$\frac{13}{41}$} & \multirow{2}{*}{$\begin{array}{c}\text { Male } \\
\text { Female }\end{array}$} & \multirow{2}{*}{$\begin{array}{c}6 \\
10\end{array}$} & \\
\hline & & & & & \\
\hline Age (years) ${ }^{a}$ & \multicolumn{2}{|c|}{$51.59 \pm 16.60$} & \multicolumn{2}{|c|}{$37.50 \pm 11.96$} & $0.00239 * *$ \\
\hline $\mathrm{SALT}^{\mathrm{b}}$ & \multicolumn{2}{|c|}{$6(2-17.5)$} & \multicolumn{2}{|c|}{$8(3-41.5)$} & 0.556 \\
\hline Body mass index $\left(\mathrm{kg} / \mathrm{m}^{2}\right)^{\mathrm{b}}$ & \multicolumn{2}{|c|}{$21.69(19.43-23.93)$} & \multicolumn{2}{|c|}{$22.28(20.35-24.53)$} & 0.321 \\
\hline Energy intake $(\mathrm{kcal} / \mathrm{day})^{\mathrm{b}}$ & \multicolumn{2}{|c|}{$1595(1326-1995)$} & \multicolumn{2}{|c|}{1789 (|22I-2047) } & 0.321 \\
\hline \multicolumn{6}{|l|}{ Nutrients } \\
\hline Animal protein $(\% \text { energy })^{b}$ & \multicolumn{2}{|c|}{$8.59(6.68-10.28)$} & \multicolumn{2}{|c|}{$9.30(7.19-10.16)$} & 0.506 \\
\hline Vegetable protein $(\% \text { energy })^{\mathrm{a}}$ & \multicolumn{2}{|c|}{$6.49 \pm 1.20$} & \multicolumn{2}{|c|}{$6.25 \pm 1.14$} & 0.485 \\
\hline Animal fat $(\% \text { energy })^{a}$ & \multicolumn{2}{|c|}{$12.44 \pm 4.39$} & \multicolumn{2}{|c|}{$14.16 \pm 4.53$} & 0.177 \\
\hline Vegetable fat $(\% \text { energy })^{\mathrm{b}}$ & \multicolumn{2}{|c|}{$15.22(13.14-18.33)$} & \multicolumn{2}{|c|}{$15.22(13.87-16.04)$} & 0.905 \\
\hline Carbohydrate (\% energy) ${ }^{a}$ & \multicolumn{2}{|c|}{$51.39 \pm 8.91$} & \multicolumn{2}{|c|}{$51.63 \pm 6.85$} & 0.921 \\
\hline $\mathrm{Na}(\mathrm{mg} / \mathrm{kcal})^{\mathrm{b}}$ & \multicolumn{2}{|c|}{$2.27(2.03-2.65)$} & \multicolumn{2}{|c|}{$2.26(2.14-2.57)$} & 0.785 \\
\hline $\mathrm{K}(\mathrm{mg} / \mathrm{kcal})^{\mathrm{b}}$ & 1.302 & & 1.255 & 44) & 0.534 \\
\hline $\mathrm{Ca}(\mu \mathrm{g} / \mathrm{kcal})^{\mathrm{b}}$ & 273.5 & & 268.2 & 7.1) & 0.498 \\
\hline $\operatorname{Mg}(\mu \mathrm{g} / \mathrm{kcal})^{\mathrm{b}}$ & 130.6 & & 114.7 & I.1) & 0.196 \\
\hline $\mathrm{Pi}(\mu g / \mathrm{kcal})^{\mathrm{b}}$ & 552.2 & & 553.3 & 6.9) & 0.764 \\
\hline $\mathrm{Fe}(\mu \mathrm{g} / \mathrm{kcal})^{\mathrm{b}}$ & 4.08 & & 4.02 & & 0.506 \\
\hline $\mathrm{Zn}(\mu \mathrm{g} / \mathrm{kcal})^{\mathrm{a}}$ & & & 4.47 & & 0.468 \\
\hline $\mathrm{Cu}(\mu \mathrm{g} / \mathrm{kcal})^{\mathrm{b}}$ & 0.582 & & 0.587 & 06) & 0.48 \\
\hline $\operatorname{Mn}(\mu g / k c a l)^{b}$ & 1.67 & & 1.57 & & $0.57 \mid$ \\
\hline Retinol $(\mu \mathrm{g} / \mathrm{kcal})^{\mathrm{b}}$ & 0.173 & & 0.197 & 306) & 0.515 \\
\hline$\beta$-carotene $(\mu \mathrm{g} / \mathrm{kcal})^{\mathrm{b}}$ & 1.923 & & 2.052 & 187) & 0.828 \\
\hline Vitamin D (ng/kcal) ${ }^{\mathrm{b}}$ & 5.512 & & 4.957 & 19) & 0.291 \\
\hline$\alpha$-tocopherol $(\mu \mathrm{g} / \mathrm{kcal})^{\mathrm{a}}$ & & & 4.1 & & 0.418 \\
\hline Vitamin $\mathrm{K}(\mu \mathrm{g} / \mathrm{kcal})^{\mathrm{b}}$ & 0.140 & & 0.115 & 58) & 0.164 \\
\hline Vitamin BI $(\mu g / k c a l)^{\mathrm{a}}$ & & & 0.4 & & 0.780 \\
\hline 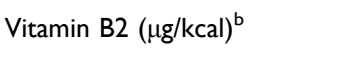 & 0.715 & & 0.722 & 364) & 0.994 \\
\hline Niacin $(\mu \mathrm{g} / \mathrm{kcal})^{\mathrm{a}}$ & & & 9.3 & & 0.463 \\
\hline Vitamin B6 $(\mu \mathrm{g} / \mathrm{kcal})^{\mathrm{b}}$ & & & 0.6 & & 0.432 \\
\hline Vitamin BI2 (ng/kcal) ${ }^{\mathrm{b}}$ & 3.91 & & 4.37 & & 0.961 \\
\hline Foric acid $(\mu \mathrm{g} / \mathrm{kcal})^{\mathrm{b}}$ & 0.180 & & 0.166 & 207) & 0.413 \\
\hline Panthotenic acid $(\mu / \mathrm{kcal})^{\mathrm{b}}$ & 3.493 & & 3.574 & 8I) & 0.883 \\
\hline
\end{tabular}

(Continued) 
Table 6 (Continued).

\begin{tabular}{|c|c|c|c|c|c|}
\hline \multirow{3}{*}{ Sex } & \multicolumn{2}{|c|}{ AA without AD $(n=54)$} & \multicolumn{2}{|c|}{ AA with AD $(n=16)$} & \multirow{3}{*}{$\frac{p \text { values }}{0.343^{c}}$} \\
\hline & \multirow{2}{*}{$\frac{\text { Male }}{\text { Female }}$} & \multirow{2}{*}{$\frac{13}{41}$} & \multirow{2}{*}{$\begin{array}{c}\text { Male } \\
\text { Female }\end{array}$} & \multirow{2}{*}{$\frac{6}{10}$} & \\
\hline & & & & & \\
\hline Vitamin C $(\mu \mathrm{g} / \mathrm{kcal})^{\mathrm{b}}$ & \multicolumn{2}{|c|}{$62.20(51.09-90.01)$} & \multicolumn{2}{|c|}{$52.74(39.0 \mathrm{I}-80.83)$} & 0.186 \\
\hline SFA (\% energy) ${ }^{\mathrm{a}}$ & \multicolumn{2}{|c|}{$7.384 \pm 1.986$} & \multicolumn{2}{|c|}{$8.311 \pm 2.309$} & 0.119 \\
\hline MUFA (\% energy) ${ }^{\mathrm{a}}$ & \multicolumn{2}{|c|}{$9.995 \pm 2.531$} & \multicolumn{2}{|c|}{$10.570 \pm 1.708$} & 0.398 \\
\hline n-3PUFA (\% energy) ${ }^{a}$ & \multicolumn{2}{|c|}{$1.333 \pm 0.396$} & \multicolumn{2}{|c|}{$1.207 \pm 0.296$} & 0.241 \\
\hline n-6PUFA (\% energy) ${ }^{a}$ & \multicolumn{2}{|c|}{$5.550 \pm 1.404$} & \multicolumn{2}{|c|}{$5.371 \pm 0.791$} & 0.627 \\
\hline Cholesterol $(\mu \mathrm{g} / \mathrm{kcal})^{\mathrm{a}}$ & \multicolumn{2}{|c|}{$203.87 \pm 74.50$} & \multicolumn{2}{|c|}{$231.06 \pm 67.23$} & 0.195 \\
\hline Dietary fiber $(\mathrm{mg} / \mathrm{kcal})^{\mathrm{b}}$ & \multicolumn{2}{|c|}{$6.476(5.059-7.918)$} & \multicolumn{2}{|c|}{$5.908(4.4 \mid 7-6.838)$} & 0.304 \\
\hline Alcohol (\% energy) $)^{b}$ & \multicolumn{2}{|c|}{$0(0-5.4 \mid 8)$} & \multicolumn{2}{|c|}{$0.537(0-2.484)$} & 0.703 \\
\hline \multicolumn{6}{|l|}{ Foods } \\
\hline Cereals $(\mathrm{mg} / \mathrm{kcal})^{\mathrm{a}}$ & \multicolumn{2}{|c|}{$\mid 81.31 \pm 68.59$} & \multicolumn{2}{|c|}{$203.74 \pm 48.28$} & 0.227 \\
\hline Potatoes $(\mathrm{mg} / \mathrm{kcal})^{\mathrm{b}}$ & \multicolumn{2}{|c|}{$15.36(9.67-28.81)$} & \multicolumn{2}{|c|}{$30.40(10.45-45.07)$} & 0.184 \\
\hline Pulses $(\mathrm{mg} / \mathrm{kcal})^{\mathrm{b}}$ & \multicolumn{2}{|c|}{$2.015(1.277-4.725)$} & \multicolumn{2}{|c|}{$2.127(1.406-3.024)$} & 0.916 \\
\hline $\begin{array}{l}\text { Green and yellow vegetables }(\mathrm{mg} / \\
\mathrm{kcal})^{\mathrm{b}}\end{array}$ & \multicolumn{2}{|c|}{$56.33(29.82-90.96)$} & \multicolumn{2}{|c|}{$58.32(38.64-78.10)$} & 0.817 \\
\hline Other vegetables $(\mathrm{mg} / \mathrm{kcal})^{\mathrm{a}}$ & & & 86.6 & & 0.794 \\
\hline Fruit $(\mathrm{mg} / \mathrm{kcal})^{\mathrm{b}}$ & 72.13 & $.11)$ & 46.38 & 47) & 0.193 \\
\hline Fish and shellfish $(\mathrm{mg} / \mathrm{kcal})^{\mathrm{b}}$ & 31.64 & 94) & 26.53 & 47) & 0.338 \\
\hline Meat $(\mathrm{mg} / \mathrm{kcal})^{\mathrm{b}}$ & 40.06 & 42) & 47.94 & 79) & 0.0974 \\
\hline Eggs $(\mathrm{mg} / \mathrm{kcal})^{\mathrm{b}}$ & 19.33 & 19) & 25.80 & 36) & 0.254 \\
\hline Dairy products $(\mathrm{mg} / \mathrm{kcal})^{\mathrm{b}}$ & 74.08 & $80)$ & 68.38 & 55) & 0.600 \\
\hline Oils and fats $(\mathrm{mg} / \mathrm{kcal})^{\mathrm{a}}$ & & & 6.75 & & 0.852 \\
\hline Confection (mg/kcal) ${ }^{\mathrm{b}}$ & 25.62 & il) & 23.22 & 28) & 0.905 \\
\hline Beverages $(\mathrm{mg} / \mathrm{kcal})^{\mathrm{b}}$ & 382.1 & I.2) & 397.5 & $5.7)$ & 0.382 \\
\hline Seasonings and spices $(\mathrm{mg} / \mathrm{kcal})^{\mathrm{b}}$ & 121.6 & & 144. I & $3.7)$ & 0.160 \\
\hline Sugar/sweeteners $(\mathrm{mg} / \mathrm{kcal})^{\mathrm{b}}$ & 2.015 & 25) & 2.127 & & 0.916 \\
\hline
\end{tabular}

Notes: ${ }^{a}$ Data provided as the mean \pm standard deviation, analyzed by Student's $t$-test. ${ }^{b} D a t a$ provided as the median (interquartile range), analyzed by Mann-Whitney U-test. 'Fisher's exact test was used to test the significance of difference in frequency distribution. $*$ Significant difference at $p<0.01$.

Abbreviations: SALT, severity of alopecia tool; SFA, saturated fatty acid; MUFA, monounsaturated fatty acid; PUFA, polyunsaturated fatty acid.

metabolite of retinol. Retinoic acid may increase the susceptibility of hair follicles to attack by NKG2D + effector cells by inducing hair follicle stem cells to enter anagen phase via signalling through the wingless-type mouse mammary tumor virus integration site family ${ }^{26}$ and by increasing the expression of NKG2D ligands such as MICA ${ }^{27}$ or MHC class I. ${ }^{28}$
Dietary retinol, which is abundant in fish, liver, eggs, and cheese, is absorbed in the intestine and delivered to the liver where it is stored as retinyl esters. Retinol released from liver stores may be delivered via the blood to the skin surrounding the hair follicles, ${ }^{29}$ And taken up by hair follicle epithelial cells or immune cells, and 
Table 7 The Association of Comorbid Atopic Dermatitis in Alopecia Areata Patients with Each Variable Analyzed by Multiple Logistic Regression Analysis

\begin{tabular}{|l|c|c|c|}
\hline & Odds Ratio & 95\% Confidential Interval & p \\
\hline (Intercept) & 1.82 & $0.0247-134.0$ & 0.785 \\
Age (years) & 0.941 & $0.901-0.984$ & $0.00743 * *$ \\
Sex $(\mathrm{M}=\mathrm{I}, \mathrm{F}=2)$ & 0.826 & $0.219-3.11$ & 0.777 \\
Body mass index $\left(\mathrm{kg} / \mathrm{m}^{2}\right)$ & 1.05 & $0.904-1.23$ & 0.502 \\
\hline
\end{tabular}

Note: **Statistically significant at $p<0.01$.

oxidized to RA. ${ }^{9,29}$ In the nucleus, RA binds to RA receptor $\alpha, \beta$, or $\gamma$, which heterodimerizes with the retinoid $\mathrm{X}$ receptor, and these dimers bind to RA response elements in various genes to activate their transcription. ${ }^{9}$ The expression levels of RA synthesis enzymes were higher in AA skin lesions than in healthy skin. ${ }^{9}$ The higher levels of dietary retinol in patients with AA may robustly promote lesional RA synthesis and RA-induced gene expression, thus exacerbating autoimmune attack.

However, RA also has immunoregulatory effects; ${ }^{30}$ it promotes the expression of Foxp3 and induces the generation of peripheral Tregs. $\mathrm{C} 3 \mathrm{H} / \mathrm{HeJ}$ AA model mice fed a vitamin A-deficient diet had more severe disease, ${ }^{9}$ which may be due to Treg defects. The balance of the stimulatory and regulatory effects of RA in AA may differ depending on the dose of RA. Further studies should elucidate the dose-dependence of the stimulatory and regulatory effects of RA on the progression of AA.

The incidence of AD in AA patients was negatively associated with age, which may reflect a general trend, irrespective of $\mathrm{AA}$, since the onset of $\mathrm{AD}$ declines with age. $^{31}$ Meat intake was higher in AA patients with AD than in AA patients without $\mathrm{AD}$, although the difference was not significant. The results might reflect the younger age of the former group, since meat consumption is associated with younger age. ${ }^{32}$ Alternatively, higher intake of fried or microwaved meats might generate higher amounts of advanced glycation end products, which function as alarmins triggering allergic diseases. ${ }^{33}$ However, the present study did not reveal any significant differences in the intake of the evaluated nutrients/foods between AA patients with $\mathrm{AD}$ and those without $\mathrm{AD}$. Further studies with larger sample sizes are needed to evaluate the involvement of dietary habits in the association between $\mathrm{AD}$ and AA.

This study has some limitations. First, the sample size was small, and the study was performed in a limited area, Chiba and Tokyo in the Kanto region of Japan. Dietary habits may vary regionally in Japan, thus the present results might not be representative of Japan as a whole. We should further extend this study to include a larger number of subjects from different regions of Japan. Second, the BDHQ calculates the intake of nutrients only from the diet and thus might overlook the intake of supplementary micronutrients. Further studies should examine all nutrient intake, including supplements. Thirdly, this study examined the dietary habits of each patient only once, and thus the correlation of dietary habits with disease course or activity is unknown. Such correlations should be examined by assessing the dietary habits of all patients several times.

\section{Conclusion}

Adult Japanese patients with AA had a higher BMI and higher intake of vitamin $\mathrm{C}$ and fruit than a group of ageand sex-matched healthy controls. AA was associated with BMI. Retinol intake was a predictor of SALT score. This is the first study to examine the dietary habits of patients with AA. Further studies should clarify whether dietary interventions to reduce the BMI or the intake of retinol can alter the development or severity of AA.

\section{Acknowledgments}

This work was partly supported by scholarship grants from Taiho Pharmaceutical, Torii Pharmaceutical, and Maruho Co., Ltd.

\section{Disclosure}

The authors report no conflicts of interest in this work.

\section{References}

1. Gilhar A, Laufer-Britva R, Keren A, et al. Frontiers in alopecia areata pathobiology research. J Allergy Clin Immunol. 2019;144:1478-1489. doi:10.1016/j.jaci.2019.08.035

2. Hong JW, Lee CY, Ha SM, et al. The contributory roles of Th17 lymphocyte and cytotoxic $\mathrm{T}$ lymphocyte at the hair bulge region as well as the hair bulb area in the chronic alopecia areata patients. Ann Dermatol. 2017;29:156-166. doi:10.5021/ad.2017.29.2.156 
3. Hamed FN, Åstrand A, Bertolini M, et al. Alopecia areata patients show deficiency of FOXP3+CD39+ T regulatory cells and clonotypic restriction of Treg TCR $\beta$-chain, which highlights the immunopathological aspect of the disease. PLoS One. 2019;14:e0210308. doi:10.1371/journal.pone.0210308

4. Suárez-Fariñas M, Ungar B, Noda S, et al. Alopecia areata profiling shows TH1, TH2, and IL-23 cytokine activation without parallel TH17/TH22 skewing. $J$ Allergy Clin Immunol. 2015;136:1277-1287. doi:10.1016/j.jaci.2015.06.032

5. Gruenstein D, Malik K, Levitt J. Full scalp hair regrowth in a 4-yearold girl with alopecia areata and atopic dermatitis treated with dupilumab. JAAD Case Rep. 2020;6:1286-1287. doi:10.1016/j. jdcr.2020.10.010

6. Thompson JM, Mirza MA, Park MK, et al. The role of micronutrients in alopecia areata: a review. Am J Clin Dermatol. 2017;18:663-679. doi:10.1007/s40257-017-0285-x

7. Manzel A, Muller DN, Hafler DA, et al. Role of "Western diet" in inflammatory autoimmune diseases. Curr Allergy Asthma Rep. 2014;14:404. doi:10.1007/s11882-013-0404-6

8. Pham CT, Romero K, Almohanna HM, et al. The role of diet as an adjuvant treatment in scarring and nonscarring alopecia. Skin Appendage Disord. 2020;6:88-96. doi:10.1159/000504786

9. Duncan FJ, Silva KA, Johnson CJ, et al. Endogenous retinoids in the pathogenesis of alopecia areata. $J$ Invest Dermatol. 2013;133:334-343. doi:10.1038/jid.2012.344

10. Rebello D, Wang E, Yen E, et al. Hair growth in two alopecia patients after fecal microbiota transplant. ACG Case Rep J. 2017;4:e107. doi:10.14309/crj.2017.107

11. Kobayashi S, Murakami K, Sasaki S, et al. Comparison of relative validity of food group intakes estimated by comprehensive and brief-type self-administered diet history questionnaires against 16 d dietary records in Japanese adults. Public Health Nutr. 2011;14:1200-1211. doi:10.1017/S1368980011000504

12. Olsen E, Hordinsky M, McDonald-Hull S, et al. Alopecia areata investigational assessment guidelines. National Alopecia Areata Foundation. J Am Acad Dermatol. 1999;40:242-246. doi:10.1016/ S0190-9622(99)70195-7

13. Olsen EA, Canfield D. SALT II: a new take on the Severity of Alopecia Tool (SALT) for determining percentage scalp hair loss. $J$ Am Acad Dermatol. 2016;75:1268-1270. doi:10.1016/j. jaad.2016.08.042

14. Kanda Y. Investigation of the freely available easy-to-use software 'EZR' for medical statistics. Bone Marrow Transplant. 2013;48:452-458. doi:10.1038/bmt.2012.244

15. Ito T. Advances in the management of alopecia areata. J Dermatol. 2012;39:11-17. doi:10.1111/j.1346-8138.2011.01476.x

16. Saeki H, Nakahara T, Tanaka A, et al. Clinical practice guidelines for the management of atopic dermatitis 2016. J Dermatol. 2016;43 (10):1117-1145. doi:10.1111/1346-8138.13392

17. Conic RRZ, Chu S, Tamashunas NL, et al. Prevalence of cardiac and metabolic diseases among patients with alopecia areata. J Eur Acad Dermatol Venereol. 2021;35:e128-e129. doi:10.1111/jdv.16864

18. Bae HR, Choi MS, Kim S, et al. IFN $\gamma$ is a key link between obesity and Th1-mediated autoimmune diseases. Int J Mol Sci. 2020;22:208 doi:10.3390/ijms22010208

Clinical, Cosmetic and Investigational Dermatology

\section{Publish your work in this journal}

Clinical, Cosmetic and Investigational Dermatology is an international, peer-reviewed, open access, online journal that focuses on the latest clinical and experimental research in all aspects of skin disease and cosmetic interventions. This journal is indexed on CAS
19. Guzik TJ, Skiba DS, Touyz RM, et al. The role of infiltrating immune cells in dysfunctional adipose tissue. Cardiovasc Res. 2017;113:1009-1023. doi:10.1093/cvr/cvx108

20. Piccio L, Cantoni C, Henderson JG, et al. Lack of adiponectin leads to increased lymphocyte activation and increased disease severity in a mouse model of multiple sclerosis. Eur J Immunol. 2013;43 (8):2089-2100. doi:10.1002/eji.201242836

21. Stochmal A, Waśkiel-Burnat A, Chrostowska S, et al. Adiponectin as a novel biomarker of disease severity in alopecia areata. Sci Rep. 2021;11:13809. doi:10.1038/s41598-021-92853-1

22. Jeong YJ, Hong SW, Kim JH, et al. Vitamin C-treated murine bone marrow-derived dendritic cells preferentially drive naïve $\mathrm{T}$ cells into Th1 cells by increased IL-12 secretions. Cell Immunol. 2011;266:192-199. doi:10.1016/j.cellimm.2010.10.005

23. Almohanna HM, Ahmed AA, Tsatalis JP, et al. The role of vitamins and minerals in hair loss: a review. Dermatol Ther (Heidelb). 2019;9:51-70. doi:10.1007/s13555-018-0278-6

24. Dai Y-X, Yeh F-Y, Shen Y-J, et al. Cigarette smoking, alcohol consumption, and risk of alopecia areata: a population-based cohort study in Taiwan. Am J Clin Dermatol. 2020;21:901-911. doi:10.1007/s40257-020-00547-7

25. Joosten MM, van Erk MJ, Pellis L, et al. Moderate alcohol consumption alters both leucocyte gene expression profiles and circulating proteins related to immune response and lipid metabolism in men. $\mathrm{Br}$ J Nutr. 2012;108:620-627. doi:10.1017/S0007114511005988

26. Suo L, Sundberg JP, Everts HB. Dietary vitamin A regulates wingless-related MMTV integration site signaling to alter the hair cycle. Exp Biol Med (Maywood). 2015;240:618-623. doi:10.1177/ 1535370214557220

27. Fan XY, Wang PY, Zhang C, et al. All-trans retinoic acid enhances cytotoxicity of CIK cells against human lung adenocarcinoma by upregulating MICA and IL-2 secretion. Sci Rep. 2017;7:16481. doi:10.1038/s41598-017-16745-Z

28. Bhattacharya N, Yuan R, Prestwood TR, et al. Normalizing microbiota-induced retinoic acid deficiency stimulates protective CD8(+) T cell-mediated immunity in colorectal cancer. Immunity. 2016;45:641-655. doi:10.1016/j.immuni.2016.08.008

29. Reichrath J, Lehmann B, Carlberg C, et al. Vitamins as hormones. Horm Metab Res. 2007;39:71-84. doi:10.1055/s-2007-958715

30. Kanda N, Hoashi T, Saeki H. Nutrition and psoriasis. Int J Mol Sci. 2020;21:5405. doi:10.3390/ijms21155405

31. Hanifin JM, Reed ML. A population-based survey of eczema prevalence in the United States. Dermatitis. 2007;18:82-91. doi:10.2310/6620.2007.06034

32. Pfeiler TM, Egloff B. Personality and attitudinal correlates of meat consumption: results of two representative German samples. Appetite. 2018;121:294-301. doi:10.1016/j.appet.2017.11.098

33. Levin ME, Botha M, Basera W, et al. Environmental factors associated with allergy in urban and rural children from the South African Food Allergy (SAFFA) cohort. $J$ Allergy Clin Immunol. 2020;145:415-426. doi:10.1016/j.jaci.2019.07.048

The manuscript management system is completely online and includes a very quick and fair peer-review system, which is all easy to use. Visit http://www.dovepress.com/testimonials.php to read real quotes from published authors. 